\title{
A l'occasion du bicentenaire d'Abraham Lincoln, inauguration d'une statue dans les jardins de l'Université de Chicago à Paris
}

Interview de l'artiste Vincent CHARRA

\section{Géraldine Chouard}

\section{OpenEdition}

\section{Journals}

Édition électronique

URL : https://journals.openedition.org/transatlantica/4553

DOI : 10.4000/transatlantica.4553

ISSN : 1765-2766

Éditeur

Association française d'Etudes Américaines (AFEA)

\section{Référence électronique}

Géraldine Chouard, «A l'occasion du bicentenaire d'Abraham Lincoln, inauguration d'une statue dans les jardins de l'Université de Chicago à Paris », Transatlantica [En ligne], 2 | 2009, mis en ligne le 01 février 2010, consulté le 01 février 2023. URL : http://journals.openedition.org/transatlantica/4553 DOI : https://doi.org/10.4000/transatlantica.4553

Ce document a été généré automatiquement le 1 février 2023.

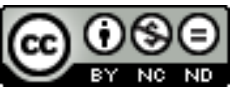

Creative Commons - Attribution - Pas d'Utilisation Commerciale - Pas de Modification 4.0 International - CC BY-NC-ND 4.0

https://creativecommons.org/licenses/by-nc-nd/4.0/ 


\title{
A l'occasion du bicentenaire d'Abraham Lincoln, inauguration d'une statue dans les jardins de l'Université de Chicago à Paris
}

\author{
Interview de l'artiste Vincent CHARRA
}

\author{
Géraldine Chouard
}

1 Le jeudi 1er octobre 2009 à 17 h00 a eu lieu au Centre à Paris de l'Université de Chicago l'inauguration d'une statue d'Abraham Lincoln dont la réalisation a été financée grâce au soutien de l'Ambassade des Etats-Unis en France, du Comité Paris Chicago et du Groupe Bensidoun. Cette statue est le produit de la collaboration artistique du mosaïcien, Vincent Charra, maître d'œuvre de ce projet, et du sculpteur Henri Marquet. Réalisée à l'occasion du bicentenaire de la naissance d'Abraham Lincoln (1809-1865), cette statue se veut un symbole de l'amitié et de la coopération entre la France et les Etats-Unis.

GC : Pourquoi une statue de Lincoln à Paris ?

VC : 2009 étant l'année du bicentenaire de naissance de Lincoln, l'Ambassade des Etats-Unis à Paris s'est émue de l'absence presque totale de monument à la mémoire de ce président américain. Elle a donc mandaté mon atelier pour y remédier.

$\mathrm{GC}$ : Comment s'est mis en place le projet?

$\mathrm{VC}$ : Le réseau universitaire des américanistes français y a fortement contribué. Lors d'échanges avec des représentants de l'Ambassade des Etats-Unis, au cours d'un colloque, Naomi Wulf, professeur d'histoire américaine, ma compagne par ailleurs, s'est fait l'écho de l'absence marquante de toute pièce commémorative de Lincoln à Paris.

Votre récente collaboration à l'exposition « Un patchwork de cultures ", relatant l'histoire américaine à travers cette pratique artistique très particulière montrait la voie. Son contenu était capital : le patchwork est en effet très proche du médium que 
j'envisageais de proposer, la mosaïque. Cette exposition a donc été une source d'inspiration décisive pour mon propre projet.

Enfin des spécialistes m'ont bien aidé à cerner le profil du président: Bernard Vincent a ainsi spontanément accepté de me recevoir et de réinscrire le parcours de Lincoln dans l'histoire des Etats-Unis.

\section{GC : Dans quelles conditions avez-vous réalisé cette statue?}

VC : Il a fallu tout d'abord réunir les fonds, préliminaire nécessaire. L'Ambassade a pris un certain nombre de contacts avec des personnes susceptibles d'assurer le mécénat d'un tel projet.

La deuxième difficulté, et non la moindre, a été de trouver un espace approprié à l'accueil d'une statue, ce qui représentait une réelle difficulté : en effet, le domaine parisien constitue pour les sculpteurs un terrain quasi impossible à conquérir, essentiellement pour des raisons de procédures administratives.

L'idée était de réaliser un monument qui allait trancher avec l'académisme souvent de rigueur quand il s'agit de commémoration. J'avais donc proposé à l'Ambassade une sculpture en mosaïque. Avec une certaine audace, Madame Lora Berg, l'attachée culturelle de l'Ambassade des Etats-Unis à Paris a accepté cette idée. Une petite enquête sur l'existence de tels monuments dans le monde a fait apparaître qu'il n'en existait pratiquement aucun. La mosaïque en tant que médium graphique a en effet presque toujours été pensée pour des œuvres en deux, et non en trois dimensions, et encore plus rarement pour de la statuaire. Et donc, sans le savoir, je me lançais dans une expérience totalement neuve, avec à la clef, un certain nombre de questions qui exigeaient également des réponses inédites.

Une fois ce projet retenu, j'ai eu la chance de rentrer en contact avec l'Université de Chicago in Paris, à côté de la BNF. Abraham Lincoln, originaire de l'Illinois et l'Université de Chicago étaient en quelque sorte faits pour se rencontrer! Madame Meltzer, directrice de UofC a donc donné son accord pour que la statue de Lincoln soit implantée dans le jardin du centre. Ce fut bien sûr une grande satisfaction que de savoir que l'œuvre en chantier allait se trouver dans un lieu aussi parfaitement adapté.

GC : D'un point de vue technique, comment s'est passée la collaboration avec le graphiste, le sculpteur, le soudeur?

VC: Nous avons la chance de former un groupe soudé avec le sculpteur Henri MARQUET, qui a à son actif de nombreuses et très imposantes réalisations dans l'espace public de Chicago.

N'étant pas moi-même sculpteur de formation, je me suis occupé de la conception graphique de la réalisation, de la mise en œuvre du projet, et, après que le corps sculpté par Henri Marquet a été fini, j'ai posé la mosaïque d'après le patron dessiné. J'ai beaucoup appris de cette expérience inédite à tous points de vue, à toutes les étapes du projet.

\section{GC : Comment voyiez-vous Lincoln avant de commencer? Pendant ? Après ?}

VC : J'avais vu le film de John Ford, Young Mr Lincoln (1939) qui donne de lui l'image d'un homme remarquable, mais peu conforme à l'idée qu'on peut se faire d'un président des Etats-Unis. Il y apparaissait comme un homme des bois, n'ayant jamais fréquenté d'autre école que celle des instituteurs itinérants et qui aimait par dessus tout raconter des histoires dans les saloons, avec un humour hors du commun. Voilà l'image que j'avais gardée de lui. Puis, la lecture de la biographie de Bernard Vincent 
m'a fait découvrir un personnage ancré dans une autre réalité. La combinaison de ces deux visons de Lincoln m'a permis de lui donner l'épaisseur charnelle dont j'avais besoin pour le sculpter.

À partir de là, j'ai surtout pris conscience du combat incroyable qui a dû se jouer dans sa conscience, le menant vers l'accomplissement de cette destinée dont le point culminant a été l'abolition de l'esclavage. Et j'ai vécu pour ainsi dire dans la peau de Lincoln pendant toute la durée du projet. Cette occupation a d'ailleurs un peu tourné à l'obsession...

GC : Lincoln a laissé dans l'histoire une image sérieuse, en tout cas moins fantaisiste que celle qui émane de son costume de céramique coloré! Pouvez-vous nous dire quelques mots sur votre interprétation du personnage?

VC : Le costume noir, austère et solennel de l'habit du président ne m'intéressait pas. Nous avions convenu avec l'Ambassade de quelque chose de plus fantaisiste, qui trancherait avec la représentation classique du personnage. L'objectif de Lincoln était de raccommoder son pays : c'est pourquoi dans l'une de mes premières maquettes, il portait un vêtement constitué de deux parties, moitié rouge, moitié bleu, un patchwork démocrate et républicain en quelque sorte !

Par ailleurs, une œuvre d'art publique doit impérativement tenir compte de l'environnement immédiat. J'ai donc rapidement opté pour des couleurs qui seraient compatibles avec la verdure du jardin où la statue allait être accueillie.

GC : Et ce «Yes we Can? » dans la manche : rappel ou projection?

VC : Bien sûr, c'est un rappel car je ne suis pas visionnaire! La filiation des deux présidents Lincoln-Obama était particulièrement stimulante, d'un point de vue visuel, car d'une certaine manière, le premier a engendré le second, ou du moins l'a rendu possible. Après avoir élaboré diverses maquettes où Lincoln prenait Obama sous son aile, un peu comme son propre enfant, la formule "Yes We Can » inscrite en carreaux de céramique dans la manche, m'a paru un raccourci satisfaisant.

\section{GC : Vouliez-vous ajouter quelque chose pour terminer?}

VC: Une dernière chose, oui. Après l'inauguration j'ai eu la surprise d'apprendre qu'un livre serait bientôt publié aux Etats-Unis recensant tous les Lincoln de 2009 : rien que dans le domaine de la sculpture, cela représente 262 œuvres! Dave Wiegers, l'auteur, m'a confié que sur ces nombreux Lincoln, le nôtre en mosaïque était quelque chose d' "unique » et a proposé de l'inclure dans son recueil. J'en suis bien sûr enchanté. Ce sera l'occasion de le faire connaitre, et qui sait, d'attirer quelques visiteurs à Paris, qui viendront faire un tour au 6, rue Thomas Mann, dans le 13ème, pour saluer le grand homme....Il les attend.

\section{INDEX}

Thèmes : Trans'Arts 
AUTEUR

GÉRALDINE CHOUARD

Université Paris-Dauphine 